\title{
Commentary: Periostin (POSTN) Regulates Tumor Resistance to Antiangiogenic Therapy in Glioma Models
}

Soon Young Park, Veerakumar Balasubramaniyan, and John F. de Groot

Department of Neuro-Oncology, The University of Texas MD Anderson Cancer Center, Houston, Texas, USA

Article Info

\section{Article Notes}

Received: February 27, 2017

Accepted: May 05, 2017

\section{${ }^{*}$ Correspondence:}

Dr. John F. de Groot, Department of Neuro-Oncology, Unit 431,

The University of Texas MD Anderson Cancer Center, 1515

Holcombe Boulevard, Houston, Texas 77030, USA. Phone:

713-745-3072; Fax: 713-794-4999, Email:

jdegroot@mdanderson.org

() 2017 de Groot J. This article is distributed under the terms of the Creative Commons Attribution 4.0 International License

\section{ABSTRACT}

Glioblastoma (GBM) are highly vascularized, invasive brain tumors with a dismal prognosis. Despite surgical de-bulking of the tumor mass followed by concomitant intensive chemo/radiotherapy, GBM patients exhibit poor survival rates (REF). The hallmark presence of microvascular proliferation in GBM has attracted vast interest in the use of antiangiogenic therapies. Bevacizumab a humanized recombinant monoclonal antibody against VEGF-A was approved by the FDA for the treatment of recurrent GBM. However, several recent studies have reported the lack of a survival benefit suggesting tumors have both intrinsic and acquired resistance to anti-VEGF therapy ${ }^{1,2}$. Studies suggest mesenchymal transition and hypoxia signaling as two major pathways associated with the development of resistance to anti-VEGF therapy. Recently, we reported that the glioma treated with bevacizumab have higher periostin (POSTN) expression than control tumors in a murine glioma tumor models ${ }^{3}$. In this commentary, we review our recent findings as well as the role of POSTN in antiangiogenic therapy resistance in glioma.

A previous study from our group found that anti-VEGF-A therapy increases invasive properties both in a GBM stem cell line (GSC11) and in glioma U87 xenograft tumors ${ }^{4}$. By using microarray analysis we found several genes including POSTN that were upregulated in bevacizumab resistant tumors. In this current study, we investigated the relationship between POSTN expression and bevacizumab resistance in mouse glioma stem cells xenograft tumors. In our model of anti-VEGF-A resistance ${ }^{3}$, GSC11 and U87 tumors treated with bevacizumab expressed higher POSTN levels than control tumors. Periostin (POSTN; osteoblastspecific factor 2) is a $90-\mathrm{kDa}$ ECM protein containing an amino-terminal EMI domain, tandem repeats of four fasciclin domains, and a carboxyterminal (C-terminal) domain, including a heparin binding site ${ }^{5}$. It has been reported that during epithelial-to mesenchymal transition (EMT), POSTN regulates several EMT markers including vimentin, fibronectin, and matrix metalloproteinase (MMP)- ${ }^{6}$. In addition to EMT markers, POSTN induces angiogenesis in endothelial cells ${ }^{7}$, and in gastric cancer cells under hypoxia conditions ${ }^{8}$. In GBM, POSTN is highly expressed when compared to normal brain ${ }^{9}$ and is associated with glioma recurrence and/or tumor progression ${ }^{10}$. Bevacizumab-resistant recurrent tumors were very large and had decreased oxygen supply in the core of the tumor as indicated by expression of the hypoxia marker HIF-1 alpha. In our study, we evaluated the potential consequence of bevacizumab on the promotion of tumor hypoxia and an aggressive glioblastoma phenotype.

We used PCR based arrays and identified POSTN regulated mesenchymal and angiogenesis signaling in glioma stem cells (GSCs). 
In our results, depletion of POSTN decreased invasion and secretion of VEGF in GSCs. Treatment of POSTN in GSCs increased invasion. Furthermore, expression of EMTrelated genes such as N-cadherin, caveolin-1 and HIF-1 alpha which were decreased after POSTN knockdown. In POSTN depleted tumors, treatment with bevacizumab, increased median survival and decreased tumor size compared to treatment with bevacizumab treatment of POSTN expressing tumors. In addition to the main source of tumor vascularization, hypoxia has an established role in the self-renewal and expansion of the GSC pool. Depletion of POSTN reduces the HIF-1 alpha expression in mouse xenograft tumors. In summary the expression of HIF-1 alpha, a key marker of resistance to anti-VEGF-A therapy, was lower in the POSTN shRNA groups than control in the xenograft glioma models.

In addition to the mechanisms mentioned above, we cannot exclude the fact that tumor microenvironmental factors also can promote GSC transdifferentiation into endothelial-like cells for the formation of new blood vessels. Importantly, GSCs can cause recruitment of immune cells, in particular, tumor-associated macrophages (TAMs) to promote angiogenesis ${ }^{11}$. We and other have shown that bevacizumab treatment increased immune cell infiltration into glioma tumors. To determine the POSTN expression in the xenografts, we used double immunofluorescence staining for the stem cell marker nestin or the macrophage marker F4/80 along with POSTN. We observed that POSTN was co-stained with nestin (tumor stem cells) but not with F4/80 (macrophage). This staining results suggest that the POSTN expression is predominant in cancer cells rather than the infiltrating macrophages. In our study, bevacizumab treatment increased TGF $\beta 1$ expression in murine GSC272 and GSC11 xenografts. However, in future studies we would need to take in account of the POSTN and their role on induction of angiogenesis via recruitment of endothelial cells and functional assays such as angiogenesis tube formation assays which will clarify the direct and indirect role of POSTN in induction of angiogenesis in glioblastoma. POSTN interacts with several integrin receptors such as $\alpha v \beta 1$ and $\alpha v \beta 3^{12}$, and it has been reported that POSTN recruits alternatively activated TAMs into the stem cell niche. Integrin $\alpha v \beta 3$ expression on monocytes and macrophages is the main receptor for POSTN-mediated TAM recruitment ${ }^{13}$. Inhibition of integrin $\alpha v \beta 3$ and integrin $\alpha v \beta 5$ prevented bevacizumab-induced invasion in orthotopic glioma models which express these integrins at high levels ${ }^{14}$. Interestingly, in our study, we found that differential expression of integrin $\beta 1$ and integrin $\beta 3$ in GSCs which play a role in cancer stem cell invasion. In particular, anti-integrin $\beta 1$ antibody inhibits invasion induced by the treatment of recombinant TGF $\beta 1$ and POSTN, whereas integrin $\beta 3$ blockade had no effect on TGF $\beta 1$ and POSTN induced invasion. Treatment of GSCs with TGF $\beta 1$ induces POSTN secretion and phosphorylation of SMAD3 and SMAD2. Furthermore, TGF $\beta 1$ increased the expression of HIF-1 alpha, VEGF-A, and STAT3 such as crucial factors involved in angiogenesis, hypoxia, and stem cell self-renewal and invasion via POSTN. However, in future studies, it will be interesting to study the combined role of POSTN and TGF $\beta 1$ in GSCs invasion and migration.. The role of TGF $\beta$ in EMT, cell survival, angiogenesis, and invasion has been well established in several solid tumors ${ }^{15,16}$. Previous studies in other organs have shown TGF $\beta 1$ induced POSTN and BMP2 during bone formation and adipocyte differentiation ${ }^{17,18}$. Both BMP2 and POSTN have been detected in glioma cell cultures, cerebrospinal fluid and brain tumor biopsy samples from glioma patients ${ }^{19}$. Recent studies have confirmed tumor stromal POSTN expression in breast cancers, melanoma ${ }^{20,21}$. In breast cancer, a small subset of metastatic cancer stem cells induce POSTN expression in the stroma of lungs for successful metastasis and colonization of lungs ${ }^{21}$. Collectively, these studies establish the role of POSTN in tumor stem cell maintenance and invasion. In GBM, tumor-associated macrophages (TAM) density positively correlates with POSTN protein levels and disrupting POSTN markedly reduced TAM density and improved survival and decreased tumor growth in a mouse GSCs xenograft model ${ }^{13}$. TGF $\beta 1$ promoted mesenchymal gene expression, especially in bevacizumab-resistant GSC cells ${ }^{22}$. In our study, we found a bidirectional signaling of TGF $\beta 1$ and POSTN in glioma stem cells and their regulation of GSCs invasion, induction of angiogenesis especially in bevacizumab-resistant GSC cells. In conclusion, in addition to TGF $\beta$ targeting, POSTN is an attractive alternative target for anti-VEGF resistant tumors, to inhibit glioma invasion, and interrupt communication between cancer stem cells and the tumor microenvironment to prevent glioma growth and progression.

\section{References}

1. Gilbert MR, Sulman EP, Mehta MP. Bevacizumab for newly diagnosed glioblastoma. N Engl J Med. 2014; 370(21): p. 2048-9.

2. Chinot $\mathrm{OL}$, et al. Bevacizumab plus radiotherapy-temozolomide for newly diagnosed glioblastoma. N Engl J Med. 2014; 370(8): p. 709-22.

3. Park SY, et al. Periostin (POSTN) Regulates Tumor Resistance to Antiangiogenic Therapy in Glioma Models. Mol Cancer Ther. 2016; 15(9): p. 2187-97.

4. Piao Y, et al. Glioblastoma resistance to anti-VEGF therapy is associated with myeloid cell infiltration, stem cell accumulation, and a mesenchymal phenotype. Neuro Oncol. 2012; 14(11): p. 1379-92.

5. Norris RA, et al. Periostin promotes a fibroblastic lineage pathway in atrioventricular valve progenitor cells. Dev Dyn. 2009; 238(5): p. 1052-63.

6. Nuzzo PV, et al. Periostin a novel prognostic and therapeutic target for genitourinary cancer. Clin Genitourin Cancer. 2014; 12(5): p. 301-11.

7. an W, Shao R. Transduction of a mesenchyme-specific gene periostin into 293T cells induces cell invasive activity through epithelialmesenchymal transformation. J Biol Chem. 2006; 281(28): p. 19700 8. 
8. Qiu F, et al. Periostin mediates the increased pro-angiogenic activity of gastric cancer cells under hypoxic conditions. J Biochem Mol Toxicol. 2013; 27(7): p. 364-9.

9. Tso CL, et al. Primary glioblastomas express mesenchymal stem-like properties. Mol Cancer Res. 2006; 4(9): p. 607-19.

10. Mikheev AM, et al. Periostin is a novel therapeutic target that predicts and regulates glioma malignancy. Neuro Oncol. 2015; 17(3): p. 37282.

11. Filatova A, Acker T, Garvalov BK. The cancer stem cell niche(s): the crosstalk between glioma stem cells and their microenvironment. Biochim Biophys Acta. 2013; 1830(2): p. 2496-508.

12. Butcher JT, et al. Periostin promotes atrioventricular mesenchyme matrix invasion and remodeling mediated by integrin signaling through Rho/PI 3-kinase. Dev Biol. 2007; 302(1): p. 256-66.

13. Zhou W, et al. Periostin secreted by glioblastoma stem cells recruits M2 tumour-associated macrophages and promotes malignant growth Nat Cell Biol. 2015; 17(2): p. 170-82.

14. Ishida J, et al. Integrin inhibitor suppresses bevacizumab-induced glioma invasion. Transl Oncol. 2014; 7(2): p. 292-302 e1.

15. Leitlein J, et al. Processing of immunosuppressive pro-TGF-beta 1,2 by human glioblastoma cells involves cytoplasmic and secreted furinlike proteases. J Immunol. 2001; 166(12): p. 7238-43.
16. Kim JE, et al. Identification of motifs for cell adhesion within the repeated domains of transforming growth factor-beta-induced gene, betaig-h3. J Biol Chem. 2000; 275(40): p. 30907-15.

17. Horiuchi K, et al. Identification and characterization of a novel protein periostin with restricted expression to periosteum and periodontal ligament and increased expression by transforming growth factor beta. J Bone Miner Res. 1999; 14(7): p. 1239-49.

18. Ji X, et al. Patterns of gene expression associated with BMP-2-induced osteoblast and adipocyte differentiation of mesenchymal progenitor cell 3T3-F442A. J Bone Miner Metab. 2000; 18(3): p. 132-9.

19. Tada T, Yabu K, Kobayashi S. Detection of active form of transforming growth factor-beta in cerebrospinal fluid of patients with glioma. Jpn J Cancer Res. 1993; 84(5): p. 544-8.

20. Kotobuki Y, et al. Periostin accelerates human malignant melanoma progression by modifying the melanoma microenvironment. Pigment Cell Melanoma Res. 2014; 27(4): p. 630-9.

21. Malanchi I, et al. Interactions between cancer stem cells and their niche govern metastatic colonization. Nature. 2011; 481(7379): p. 85-9.

22. Piao Y, et al. Acquired resistance to anti-VEGF therapy in glioblastoma is associated with a mesenchymal transition. Clin Cancer Res. 2013; 19(16): p. 4392-403 